\title{
AS BARRAS DE CALCULAR DE NAPIER: PERCEPÇÕES DE UMA PRIMEIRA MANIPULAÇÃo
}

\section{NAPIER'S CALCULAR BARS: PERCEPTIONS OF A FIRST MANIPUATION}

\author{
Eugeniano Brito Martins ${ }^{1}$ \\ Instituto Federal do Ceará - IFCE Campus Canindé \\ Ana Carolina Costa Pereira ${ }^{2}$ \\ Universidade Estadual do Ceará
}

\begin{abstract}
Resumo
Este artigo apresenta um recorta da pesquisa de mestrado sobre a manipulação das barras de calcular que John Napier descreveu no tratado Rabdologiae de 1617. Objetivando a identificação de potencialidades didáticas que venham a construir interfaces entre a história da matemática e o ensino. A forma como desenvolvemos essas potencialidades é dentro de um processo de compreensão histórico cultural da época em que o tratado foi escrito e como o ator foi influenciado durante todo o processo. Fazemos uma rápida apresentação do tratado e do conteúdo, com destaque para a parte que iremos tratar no artigo. Trata-se de uma pesquisa bibliográfica, participativa. No tratado escolhemos um dos instrumentos contidos neles para o desenvolvimento de atividades baseada nas orientações para a utilização do instrumento. Estas foram aplicadas aos participantes de um curso de extensão universitária, que foram observados e tiveram suas opiniões e impressões registradas para posterior análise. Das práticas realizadas, descrevemos aplicação de uma prática delas, a que faz a apresentação do instrumento, conforme as orientações de John Napier (1550-1617) para sua construção e preenchimento. Apresentamos a impressões destes participantes ao primeiro contato com o instrumento e como eles descreveram a mobilização de conceitos matemáticos. Concluindo que o instrumento possui diversas outras potencialidades didáticas, além da habitualmente utilizada, que é multiplicação.
\end{abstract}

Palavras-chave: Barras de Calcular; Potencialidades didáticas; Rabdologiae.

\section{Abstract}

This article presents a clipping from the master's research on the manipulation of the calculus bars described by John Napier in the treatise Rabdologiae of 1617. Aiming to identify didactic potentialities that will build interfaces between the history of mathematics and teaching. The way we develop these potentialities occurs within a process of historical-cultural understanding of the time the treatise was written and how the author was influenced throughout the process. We made a brief presentation of the treatise and the content, highlighting the part we will deal with in the article. It is a bibliographic, participatory research. In the treatise we chose one of the contained

\footnotetext{
${ }^{1}$ E-mail: eugeniano.martins@ifce.edu.br.

${ }^{2}$ E-mail: carolina.pereira@uece.br.
} 
instruments and the guidelines for its construction and manipulation, for the development of the activities. These were applied to the participants of a university extension course, who were observed and had their opinions and impressions recorded for later analysis. From the practices performed, we describe the application of one of them, which makes the presentation of the instrument, according to the guidelines of John Napier (15501617), for its construction and completion. We present the impressions of these participants upon first contact with the instrument and how they described the mobilization of mathematical concepts. Concluding that the instrument has several other didactic potentialities, besides the usual one, that is for the accomplishment of the multiplication operation.

Keywords: Napier's bars; Didactic potentialities; Rabdologiae.

\section{Introdução}

Para ensinar matemática, sabemos que não basta apenas o domínio dos conceitos formais dessa ciência. Além disso, são necessários conhecimentos referentes à didática, pedagogia, filosofia, epistemologia, dentre outros, possibilitando, assim, relacionar a realidade social dos alunos e professores com os conteúdos ensinados.

Em estudos recentes, como os de Pereira e Saito (2018, 2019), uma abordagem, que articula as áreas de história da matemática e a educação matemática, vem sendo discutida, especificamente, na formação de docentes, com vista a associar conceitos formais a outros didáticos e pedagógicos (PEREIRA; SAITO, 2019). Mesmo sabendo que a história da matemática não é um recurso didático que seja aplicado em todas as situações e que o docente tem um currículo a lecionar (FRIED, 2001), compreendemos que sua inserção tem muito a contribuir para o ensino dessa área.

Atinente a isso, este recorte da nossa pesquisa apresenta uma tentativa de articulação da história da matemática com o ensino, possibilitando o reconhecimento da matemática como uma criação humana, proveniente de resultados das necessidades práticas de comunidades (D’AMBROSIO, 2000; MIGUEL; MIORIM, 2004).

Uma forma de inserir a história da matemática em sala de aula está nas pesquisas que buscam a articulação dessa com o ensino da matemática, criando-se, assim, interfaces que conectam as duas áreas. Desde a década de 1980, diversos autores brasileiros, tais como Mendes (2009), Silva (2013), Pereira e Pereira (2015), Chaquiam (2017), dentre outros, desenvolveram pesquisas e publicaram livros com essa abordagem.

Uma das formas de realizar essa articulação, entre a história e o ensino da matemática, é através da construção e/ou a manipulação de instrumentos históricos. Para 
a utilização desses recursos, alguns estudos os associam com a escolha de um texto em que a sua construção e utilização são descritas (PEREIRA; SAITO, 2018, 2019).

Porém, para que haja a integração da história da matemática com o ensino dos diferentes conteúdos, o docente que leciona matemática necessita que sua formação, inicial ou continuada, tenha-o preparado para articular essas duas áreas de conhecimentos (BARONI; TEXEIRA; NOBRE, 2009). Desse modo, essa formação possibilita ao docente de matemática conhecimento para articular a história com o ensino. ${ }^{3}$

Diferentemente, nesta pesquisa, realizamos uma tentativa de articular a história com o ensino de matemática baseada em uma historiografia atualizada ${ }^{4}$, diferindo portanto, daquelas baseadas em uma historiografia tradicional ${ }^{5}$. Dessa maneira, buscamos a mobilização dos conhecimentos de uma época passada, sem retirar do contexto em que foi criada, construída ou aplicada, para a compreensão de conteúdo ou conceitos lecionados e que estão baseados naquele conhecimento.

Esse diálogo pode emergir de um documento histórico ou de um fragmento dele, de um instrumento, de um monumento, de uma foto, de uma imagem, entre outros (PEREIRA; SAITO, 2018). Isto é, aquilo que o historiador identifica como fonte histórica e o educador matemático, por sua vez, identifica como um objeto matemático.

Escolhemos, para esta pesquisa, o tratado Rabdologiae, Seu Numerationis Per Virgulas Libri Duo: cum appendice de expeditíssimo Multiplicationes promptuario, quibus acessit e arithmeticea localis liber $u_{n u s}{ }^{6}$, de 1617, escrito pelo escocês John Napier (1550-1617). Essa edição foi impressa em latim medieval, por Andreas Hart, tipógrafo que já tinha publicado outras obras do autor.

Do tratado, retiramos alguns excertos, que após um tratamento didático, visando a adequação do texto para a linguagem atual, foram utilizados em um curso de extensão universitária com professores em formação, todos eles alunos da licenciatura em matemática da Universidade Estadual do Ceará - UECE, que será descrito em maiores

\footnotetext{
${ }^{3}$ Para maiores informações, veja: Mendes (2009); Saito e Dias (2011); Silva (2013); Pereira e Pereira (2015); Saito (2015); Chaquiam (2017).

4 “A Historiografia atualizada procura partir do passado em direção ao presente na medida que é a partir de um acontecimento do passado que se deve entender o presente e não o contrário" (SAITO, 2015, p 27).

5 "Na historiografia tradicional o passado é visto com os olhos de hoje. Admite-se que a ciência teria se desenvolvido progressiva e linearmente. Nessa perspectiva, a História da Ciência representaria o progresso do espírito humano e da sociedade" (SAITO, 2014, p. 20).

${ }^{6}$ Ao longo do texto o nome completo da obra será referenciado como Rabdologiae (1617).
} 
detalhes adiante. Visto que, antes, é necessário conhecermos o tratado, as razões da escolha e sua importância histórica.

\section{Conhecendo um pouco o tratado Rabdologiae (1617)}

Os instrumentos matemáticos ${ }^{7}$ partem de um momento histórico em que emergiu um grande desenvolvimento social, político e econômico em países europeus, como a Inglaterra, Portugal, Escócia e outros. Eles faziam parte da necessidade de uma maior precisão na obtenção de medidas e cálculos e a descrição de suas construções se encontram presentes em tratados elaborados para atender a um público específico.

Entre esses tratados, temos o Rabdologiae, (1617), escrito por John Napier (15501617). Sua obra aborda um instrumento conhecido como as barras de calcular de Napier, explicando como construir e manusear tal objeto para a realização das operações de multiplicação, divisão, potenciação e radiciação.

O tratado que analisamos, nesta pesquisa, foi escrito em 1615. Percebemos, ao longo do texto, que o autor cita esse ano em diversos exemplos, porém ele só foi publicado em 1617. Como muitas das obras da época, seu título apresenta os conteúdos que o leitor encontrará em seu interior, assim temos: "Os dois livros de calcular com pequenas varetas" (Rabdologiae, seu numerationis per virgulas libri duo).

Nesse período, o título de um tratado apresentava uma descrição dos conteúdos presentes em suas páginas. Napier (1617) não foge à regra e apresenta, no título da obra, uma forma de realizar cálculos com menos tempo. No entanto, a invenção desses instrumentos não foi feita com essa finalidade, como apresentado por Rice e GonzálesVelasco (2017, p. 49, tradução nossa):

Como parte do cálculo de suas tabelas logarítmicas, Napier inventou uma série de ferramentas de cálculo que ele descreveu na Rabdologia. Inicialmente Napier não considerou nenhum deles digno de serem publicados, mas tendo compartilhado suas invenções com seus amigos, eles estavam se tornando conhecidos na Escócia e no exterior e corriam o risco de serem apropriados por outros. ${ }^{8}$

\footnotetext{
${ }^{7}$ Ver Saito (2015).

${ }^{8}$ No original, lemos: As part of the computation of his logarithmic tables, Napier invented a number of calculating tools which he described in the Rabdologia. Initially Napier had not considered any of them worth publishing but having shared his inventions with his friends, they were becoming known both in Scotland and abroad and were in danger of being appropriated by others (RICE; GONZÁLES-VELASCO, 2017, p. 49).
} 
Observamos, pelo trecho da dedicatória, que o objetivo de Napier era substituir os cálculos com números naturais, que ele considerava complicados e difíceis de serem realizados, e passar a utilizar os logaritmos. Logo, ele criou alguns instrumentos que facilitaram os cálculos para a construção das tabelas logarítmicas. Napier (1617) cita as duas razões principais da publicação desse tratado:

\footnotetext{
Eu tinha duas razões para fazer o meu livro, sobre a fabricação e uso das barras de calcular, disponíveis ao público. A primeira foi a de que as barras foram bem aceitas por tantas pessoas que quase se podia dizer que já eram de uso comum, tanto na Escócia, como no exterior. A outra razão foi o fato de terem chamado a minha atenção e que gentilmente me aconselhou a publicá-los, para que não sejam publicados sob o nome de outra pessoa (NAPIER, 1617, f. 3, tradução nossa) ${ }^{9}$
}

Aparentemente, Napier não iria divulgar seus inventos, que fizera para facilitar os cálculos dos logaritmos, só o fazendo por insistência do amigo Alexander Seton e para assegurar que seu nome estaria ligado às invenções que realizara.

Observamos que os instrumentos de Napier são para uso com os números naturais, objetivando facilitar o enfadonho processo de realização dos cálculos (NAPIER, 1617). Então, a partir da preocupação com a necessidade de realizar cálculos mais rápidos e com precisão, o tratado apresenta a construção e a utilização dos três instrumentos de cálculos.

$\mathrm{O}$ instrumento apresentado neste artigo é denominado de barras de calcular de Napier (Figura 1). As barras de calcular de Napier estão apresentadas ao longo de 42 fólios, divididos em nove capítulos no Livro Primeiro, no qual, em dois capítulos, são descritos sua fabricação e preenchimentos e, nos sete seguintes, sua utilização para realizar as operações de multiplicação, divisão, extração de raízes quadradas e cúbicas e na resolução de regras de três simples e compostas.

Os instrumentos matemáticos existentes na época focavam na aplicação dos conceitos matemáticos sem se descuidarem dos aspectos formais. Assim, o tratado Rabdologiae (1617) é uma das obras que se enquadra nesses aspectos, ao mobilizar conceitos matemáticos para instrumentos que auxiliavam nos cálculos aritméticos.

\footnotetext{
${ }^{9}$ No original, lemos: Ut autem libellum de Fabrica \& vsus virgularum publici juris facerem, hoc imprimis impulit, quod eas non folum viderem permultis ita placuiffe, ut jam fere fint vulgares, \& in exteras etiam regiones deferantur: fed perlatum quoque fit ad aures meas humanitatem tuam mihi confuluiffé utid ipfum facerem (NAPIER, 1617, f. 3).
} 


\section{Figura 1 - Representação das Barra de calcular de Napier}

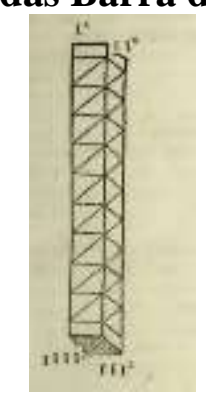

Fonte: Napier (1617, f.3).

Nessa compreensão, as barras de calcular de Napier incluem a construção e a manipulação de conhecimentos matemáticos, que facilitam a realização das operações de multiplicações e divisão, como Maor (2006, p. 15, apresenta:

Percebendo que não há nada mais trabalhoso na prática matemática, nem que mais prejudique e atrapalhe os calculadores, do que as multiplicações, as divisões, as extrações do quadrado e do cubo dos números muito grandes ... comecei a considerar em minha mente através de que tipo de arte certa e rápida poderia remover essas dificuldades.

O fato de Napier (1617) ter especial atenção à aplicabilidade da matemática foi o que motivou a articulação entre as barras de calcular e o ensino de Aritmética, buscando identificar potencialidades didáticas presentes na obra Rabdologiae (1617). Para isso, adotamos a proposta de Saito (2015), que consiste na compreensão do objeto matemático histórico com base nos conhecimentos da época de sua produção, evitando-se, portanto, que os conceitos matemáticos identificados sejam descaracterizados do real contexto em que foram concebidos, descrevendo-os de forma anacrônica e progressista.

Com base nisso, a escolha do documento, que será utilizado na construção da interface, deve ser criteriosa, pois dificilmente ele poderá ser inserido em uma atividade didática sem que as barreiras, da língua em que foi escrito, da época de sua elaboração e de sua finalidade, passem inicialmente por um tratamento que propiciem sua adequação. Logo, apresentamos a seguir as atividades desenvolvidas.

\section{A Atividade desenvolvida baseada no Rabdologiae (1617)}

Uma das finalidades da pesquisa foi compreender como os participantes de um curso de extensão mobilizariam os conhecimentos matemáticos presentes nas barras de 
calcular de Napier. Para isso, elaboramos algumas atividades, sendo que uma delas será apresentada aqui e terá seus resultados comentados.

O curso de extensão foi concretizado em parceria com a Pró-Reitora de Extensão da UECE, com o Grupo de Pesquisa em Educação e História da Matemática (GPEHM), com o Laboratório de Matemática e Ensino da UECE e com a Pós-Graduação em Ensino de Ciências e Matemática (PGECM) do Instituto Federal de Educação, Ciência e Tecnologia do Ceará (IFCE). Estando aprovado pelo conselho de ética na pesquisa da Universidade Estadual do Ceará, sob CAAE 08268319.7.0000.5534 e parecer de número 3.285.665.

O curso de extensão contou com 8 participantes, tendo sido realizado durante 4 manhãs consecutivas. Os participantes trabalharam em equipes fundamentadas na definição de trabalho em grupo, dado por Cohen e Lotan (2017, p. 1-2): “alunos trabalhando juntos em grupos pequenos de modo que todos possam participar de uma atividade com tarefas claramente atribuídas".

Para a realização do curso, foram providenciados os seguintes materiais: quatro conjuntos de 10 barras de calcular de Napier (Figura 2), conforme descrito no tratado. Cada atividade era composta de um cartão de recurso, que continha um excerto da obra; um cartão de atividades que instruíam os participantes a elaborar um relatório final por cada cartão; cartão de hipóteses englobando todas as conjecturas elaboradas para as atividades do curso.

\section{Figura 2 - Um conjunto de 10 barras de calcular de Napier.}

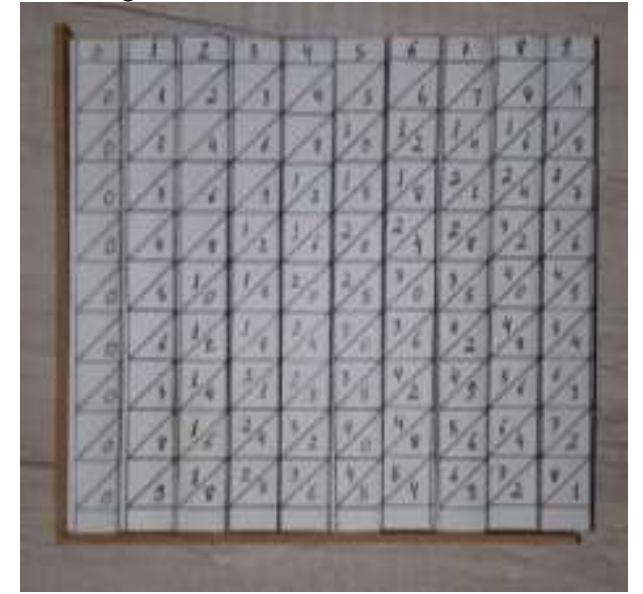

Fonte: Elaborada pelo autor (2018). 
Neste artigo, apresentaremos a prática que consistiu no contato inicial dos participantes com as barras de calcular de Napier, de acordo com o descrito no tratado. $\mathrm{O}$ objetivo dessa prática foi compreender o instrumento, sua construção e os conhecimentos matemáticos presentes na sua elaboração.

Para a realização dessa prática, foram elaborados e disponibilizados aos participantes materiais de apoio, tais como: a reconstrução de quatro conjuntos de 10 barras de calcular de Napier (Figura 2), consoante o descrito na obra, um cartão de recursos e um cartão de atividade (Quadro 1). Além disso, os participantes tiveram sobre suas mesas: lápis, canetas, borracha, papel ofício. Dessa maneira, a prática foi composta pelo capítulo I, do livro primeiro Rabdologiae (1617), no qual é apresentada a construção e como preencher os números nas barras de calcular.

\section{Quadro 1: Cartão de Atividades da Prática}

\section{CARTÃO DE ATIVIDADES - PRÁTICA}

A partir do instrumento (físico) e do texto que traz a descrição do uso das barras de calcular de John Napier:

- $\quad$ Acompanhe e entenda no que consiste o instrumento e procure reconhecer e mapear os diferentes conhecimentos matemáticos nele incorporado.

- $\quad$ Compreenda o funcionamento do instrumento.

\section{Produto do grupo - ATIVIDADE}

Anote suas primeiras impressões sobre as partes e o funcionamento do instrumento, a partir da construção e manipulação.

Fonte: Elaborada pelo autor.

O excerto do tratado recebeu o tratamento didático, que consistiu na versão em língua portuguesa e no ajuste dos termos e da forma de escrita para adequarmos à forma habitual como hoje são expressos alguns termos, evitando-se, por conseguinte, a ocorrência de dificuldades que viessem a prejudicar a realização da atividade dessa prática (SAITO; DIAS, 2013), constituindo o cartão de recursos da mesma, os fólios de 1 a 5 do tratado.

A atividade teve como objetivo orientar os participantes para que, durante o processo de conhecimento das barras de calcular e da maneira como fabricá-la, fossem identificados os conhecimentos matemáticos presentes ao longo do texto, na fabricação e no preenchimento. 


\section{Os resultados da Prática 1}

Nessa prática, os participantes receberam o excerto da obra que trata sobre a construção das barras, denominado de cartão de recurso (Anexo), que continha a descrição da fabricação do instrumento. Além disso, eles tiveram acesso a uma reconstrução das barras de calcular de Napier, composta por um conjunto de 10 barras, para ilustrar a leitura.

Durante a prática os participantes, dois observadores, registravam as suas impressões por escrito, além de estarem sendo gravados e filmados. Dessa forma, conseguimos os registros, relacionados ao primeiro contato com as barras de calcular de Napier. Apresentamos a seguir alguns registros:

- [...] para a construção do instrumento Napier utilizou conceitos geométricos, dentre eles, ângulos, proporção, diagonais.

- [...] é importante ressaltar a ordem como são dispostas as ordens das faces na barra e como os números são distribuídos entre as aréolas, sem a unidade posta na aréola inferior e a dezena na superior ( $\mathrm{sic}$ ).

- É interessante a ordem em que é posicionada os números, de maneira que, ao somar com o 'seu oposto', resultará sempre em uma múltiplo de 9, sendo a $1 \underline{a}$ face com a $3 \underline{a}$ e a $2^{-}$com a $4^{a}$, de acordo com as posições dos quadrados, o oposto é classificado na rotação da barras em um ângulo de $180^{\circ}$ em torno do seu próprio eixo (sic).

Percebemos, nas falas, a necessidade da manipulação das barras como parte do processo de construção. Pois, dessa forma, eles visualizaram a incorporação dos conhecimentos matemáticos presentes nas faces das barras para compreender sua manipulação e só, posteriormente, a construção.

A forma de dispor as barras de calcular de Napier parece estar relacionada à manipulação, já que aparentemente eles imaginaram uma disposição espacial para que elas pudessem ser utilizadas. (Figura 3)

Figura 3 - Primeira disposição das barras de calcular de Napier.

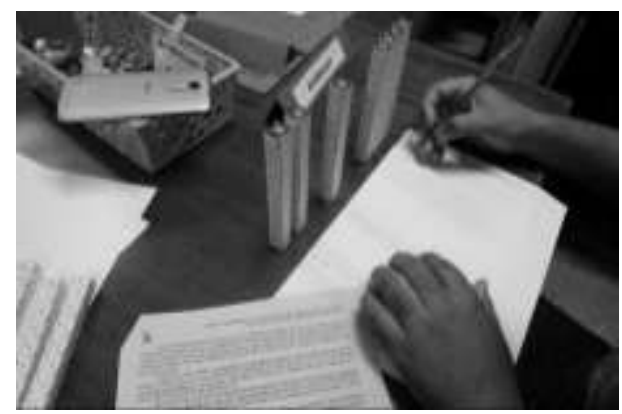

Fonte: Arquivo pessoal do autor (2018) 
O Grupo conseguiu mobilizar as denominações atuais (unidade e dezena) para a descrição da fabricação das barras de calcular de Napier, quando o autor se refere ao "número simples e o que fica à esquerda" (NAPIER, 1617, f. 10). Mesmo apresentando, de forma didática, a ordem posicional, não são utilizadas no tratado as denominações que temos atualmente

Os apontamentos também mostram que eles compreenderam que Napier (1617) preencheu as barras de modo a dispor os valores do topo de uma face como a diferença do resultado de 9 menos o valor do topo da face oposta. Contudo, não apresentaram uma razão plausível para essa forma de representação.

\section{Considerações finais}

As tendências metodológicas para o ensino de matemática apresentam diferentes recursos para o professor lecionar os conteúdos. Uma dessas tendências é a história da matemática, que pode ser utilizada como recurso didático de diferentes formas. Assim, a utilização de uma historiografia atualizada, para promover o diálogo, a história e o ensino da matemática, pode gerar atividades com potenciais pedagógicos que motivem os alunos a compreenderem os conceitos estudados.

O fato de entender o momento histórico e contextual e como ele influencia no aspecto epistemológico, para a mobilização de conhecimentos matemáticos de uma época, ao mesmo tempo em que possibilita um diálogo com o ensino para a identificação de potencialidades didáticas no passado que contribuam no presente, foi a motivação para esta tentativa de realizar esse diálogo com a história, presente no tratado Rabdologiae (1617), e o ensino da matemática.

Nessa forma de utilizar a história, observamos o passado desprovido das concepções atuais. Assim, ao explorar os conceitos matemáticos presentes no tratado, fazemo-lo respeitando a forma como foram aplicados pelo autor. A elaboração do diálogo, entre o momento histórico e o ensino, ocorre na possibilidade de mobilizarmos as ideias matemáticas observadas, de modo a contribuir com o ensino da matemática.

Ao incitarmos os diferentes conhecimentos matemáticos com a manipulação das barras de calcular de Napier, como descrito no tratado Rabdologiae (1617), durante o 
curso de extensão universitária, percebemos, nas falas dos participantes, a possibilidade de utilizar o instrumento para o desenvolvimento de atividades didáticas para o ensino de matemática.

\section{Referências}

BARONI, R. L. S.; TEIXEIRA, M. V.; NOBRE, S. R. A investigação científica em História da Matemática e suas relações com o programa de pós-graduação em Educação Matemática. In: BICUDO, M. A. V.; BORBA, M. C. Educação Matemática: pesquisa em movimento. 3 ed. São Paulo: Cortez, 2009.

CASTILLO, A. R. M. Um estudo sobre os conhecimentos matemáticos incorporados e mobilizados na construção e no uso do báculo (cross-staff) em A Boked Named Tectonicon de Leonard Digges. 2016. 121 f. Tese (Doutorado) - Curso de Educação Matemática Matemática, Pontifícia Universidade Católica de São Paulo, São Paulo, 2016.

CHAQUIAM, M. Ensaios temáticos: História da matemática em sala de aula. Belém: Sociedade Brasileira de História da Matemática, 2017. 241 p.

COHEN, E. G.; LOTAN, R. A. Planejando o trabalho em grupo. 3. ed. Porto Alegre: Penso, 2017. Tradução de: Luís Fernando Marques Dorvillé, Mila Molina Carneiro, Paula Márcia Schmaltz Ferreira Rozin.

D'AMBROSIO, U. A interfase entre História e Matemática: uma visão históricopedagógica. In: FOSSA, John A. Ensaios sobre Educação e História da Matemática. Rio Claro: Sbhmat, 2000. p. 241-271.

FRIED, M. N. Can Mathematics Education and History of Mathematics Coexist? Science And Education, [s.1.], v. 10, n. 4, p.391-408, jul. 2001. Springer Nature.

MAOR, Ei. e: A História de um Número. Lisboa: Gradiva, 2006. 268 p.

MENDES, I. A. Investigação Histórica no Ensino da Matemática. Rio de Janeiro: Ciência Moderna Ltda, 2009.

MIORIM, M. A.; MIGUEL, A. História na Educação Matemática: Proposta e Desafios. Belo Horizonte: Autêntica, 2004. (Coleção Tendências em Educação Matemática, n. 10).

NAPIER, J. Rabdologiae, Seu Numerationis Per Virgulas Libri Duo: cum appendice de expeditíssimo Multiplicationes promptuario, quibus acessit e arithmeticea localis liber unus. Edinburgh: Andrew Hart, 1617. 
PEREIRA, A. C. C.; PEREIRA, D. E. Ensaio sobre o uso de fontes históricas no ensino de matemática. Rematec: Revista de Matemática, Ensino e Cultura, Natal, v. 10, n. 18, p.65-78, jan/abr. de 2015.

PEREIRA, A. C. C.; SAITO, F. A reconstrução do báculo de Petrus Ramus na interface entre história e ensino de matemática. Revista Cocar, Belém, v. 25, n. 13, p.342-372, Jan./Abr., 2019.

PEREIRA, A. C. C.; SAITO, F. Os instrumentos matemáticos na interface entre história e ensino de matemática: compreendendo o cenário nacional nos últimos 10 anos. In: SEMINÁRIO CEARENSE DE HISTÓRIA DA MATEMÁTICA, 3., 2018, Fortaleza. Anais... . Fortaleza: Eduece, 2018. p. 1 - 12.

RICE, B.; GONZÁLEZ-VELASCO, E. e CORRIGAN, A. The Life and Works of John Napier. Switzerland: Springer, 2017. 994 p.

SAITO, F. Construindo interfaces entre história e ensino da matemática. Ensino da Matemática em Debate, v. 3, n. 1, 2016, p. 3-19.

SAITO, F. História da matemática e suas (re)construções contextuais. São Paulo: Editora Livraria da Física, 2015. 259 p.

SAITO, F. Instrumentos matemáticos dos séculos XVI e XVII na articulação entre história, ensino e aprendizagem de Matemática. Rematec: História de Práticas Matemáticas, Natal, v. 16, n. 9, p.25-47, maio/ago. 2014.

SAITO, F.; DIAS, M. da S. Articulação de entes matemáticos na construção e utilização de instrumentos de medida do século XVI. Natal: Sociedade Brasileira de História da Matemática, 2011. 63 p.

SAITO, F.; DIAS, M. da S. Interface entre história da matemática e ensino: uma atividade desenvolvida com base num documento do século XVI. Ciênc. educ. (Bauru), Bauru , v. 19, n. 1, p. 89-111, 2013. 